\title{
Invasive Broncho-Pulmonary Mucormycosis (IBPM) Misdiagnosed as Tuberculosis
}

\author{
Shital Patil ${ }^{1 *}$ (1), Rajesh Patil ${ }^{1}$ (1)
}

${ }^{1}$ MIMSR Medical College Latur, Maharashtra, INDIA

*Corresponding Author: drsvpatil1980@gmail.com

Citation: Patil S, Patil R. Invasive Broncho-Pulmonary Mucormycosis (IBPM) Misdiagnosed as Tuberculosis. ELECTR J MED DENT STUD. 2022;6(1):em0094. https://doi.org/10.29333/ejmds/11562

\section{ARTICLE INFO}

Received: 20 Oct. 2021

Accepted: 1 Dec. 2021

\begin{abstract}
Hemoptysis is nonspecific respiratory symptom with variable etiological factors ranging from infections like tuberculosis, pneumonia, fungal infections to malignant lung process. Although Tuberculosis is leading cause of hemoptysis and reason for empirical treatment and delay in diagnosis, computerized tomography and fiberoptic bronchoscopy has made real change towards approach in management of these cases presented with nonspecific symptoms. In this case report, we have documented 'invasive bronchopulmonary Mucormycosis' as cause of recurrent hemoptysis and persistent pulmonary opacity on chest radiograph irrespective of best possible conventional treatment including higher antibiotics and blanket cover of anti-tuberculosis treatment for 4 months. We have documented 'Reverse halo sign' on chest CT imaging and final diagnosis established with Bronchoscopy guided lung biopsy showing 'presence of fungal infection causing extensive parenchymal necrosis, angioinvasion and cartilage destruction along with bronchiolar invasion.' Caution should be taken during biopsy of these hypervascular lesions as propensity to have post-biopsy 'catastrophic massive hemoptysis.' We have also documented near total complete resolution of airway and lung lesions to medical management including Amphotericin and Posaconazole.
\end{abstract}

Keywords: invasive broncho-pulmonary mucormycosis, tuberculosis, reverse halo sign, CT thorax

\section{INTRODUCTION}

Mucormycosis is a rare opportunistic fungal infection. It derives its name from the Mucorales order of filamentous fungi and family Mucoraceae. It is a less common opportunistic fungal infection compared to Candida and Aspergillus species [1]. There are six most commonly reported forms which include rhinocerebral, pulmonary, gastrointestinal, cutaneous, disseminated, and uncommon presentations [2,3]. The relative incidence of pulmonary mucormycosis (PM) to other clinical form incidence in literature is about 25\% [4]. Diabetes mellitus, systemic corticosteroid therapy, neutropenia, hematologic malignancies, stem cell transplant, and immunocompromised state are the predisposing situations for Mucormycosis [3,4]. Pulmonary mucormycosis results from the inhalation of sporangiospores or by hematogenous or lymphatic spread [3,5]. Patients present with nonspecific symptoms like cough, dyspnea, chest pain, and fever [3,5]. Clinical diagnosis is difficult in pulmonary mucormycosis, and early diagnosis is needed for this lifethreatening infection [6]. There are no reliable serological, PCR-based, or skin tests for mucormycosis. Sterile culture does not rule out the diagnosis. Histopathology and direct microscopy along with culture from various clinical specimens except blood are the major diagnostic modalities for Mucormycosis [5]. In this case report, we have documented empirical anti-tuberculosis is an absolute option now and bronchoscopy has very crucial role in diagnosing unexplained hemoptysis and bronchoscopy guided techniques will be crucial during entire course of illness from diagnosis to management of pulmonary Mucormycosis cases.

\section{CASE SUMMARY}

43-year male, driver by occupation, alcohol and tobacco addict, newly diagnosed uncontrolled diabetes mellitus, presented with history of recurrent hemoptysis, dry cough since 4 months, received antituberculosis treatment at general physician center with Isoniazid, Rifampicin, Ethambutol, and Pyrazinamide for first 2 months and Isoniazid, Rifampicin, and Ethambutol for next 2 months. He was diagnosed as pulmonary tuberculosis on basis of chest x-ray and sputum examination was inconclusive and sputum gene Xpert MTB/RIF were negative done by general physician. Patient is referred to our center for recurrent hemoptysis and radiological worsening. 
His first chest $\mathrm{x}$-ray done at general physician center was showing right upper lobe inhomogeneous opacification (Figure 1). He was admitted there, investigated as sputum microscopy for acid fast bacilli and sputum Gene Xpert MTB/RIF and results were inconclusive and negative for mycobacterium tuberculosis genome. He has been started on antibiotics, give for 10 days and empirical anti-tuberculosis treatment were started. After one month of anti-tuberculosis treatment, he was having intermittent hemoptysis with persistent cough, repeated chest $\mathrm{x}$-ray which was showing increased right upper zone opacity, i.e., Radiological worsening (Figure 2).

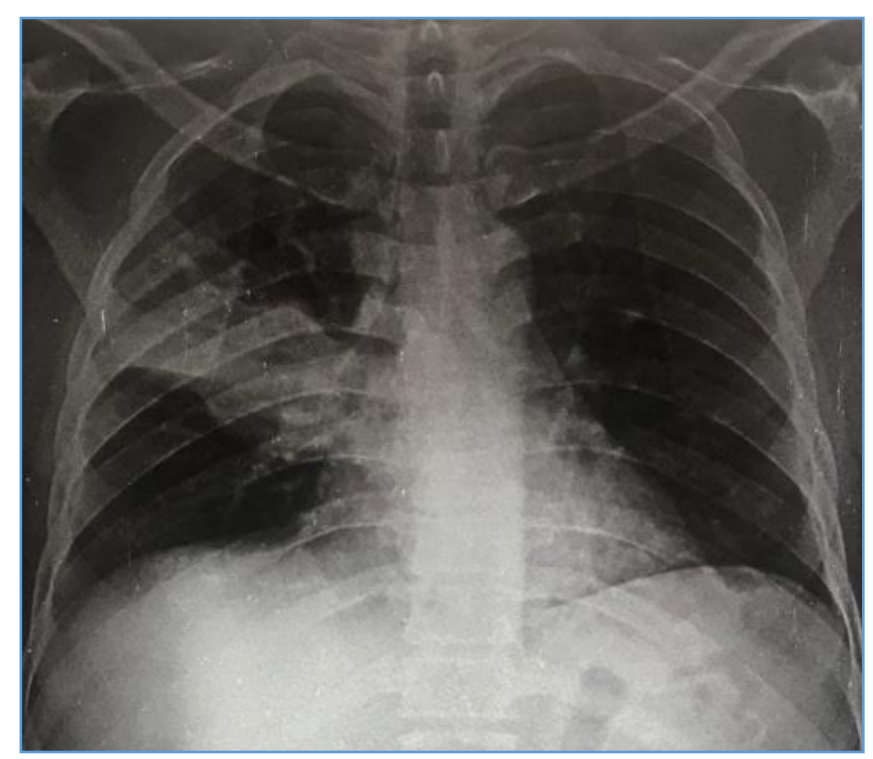

Figure 1. Chest radiograph-1

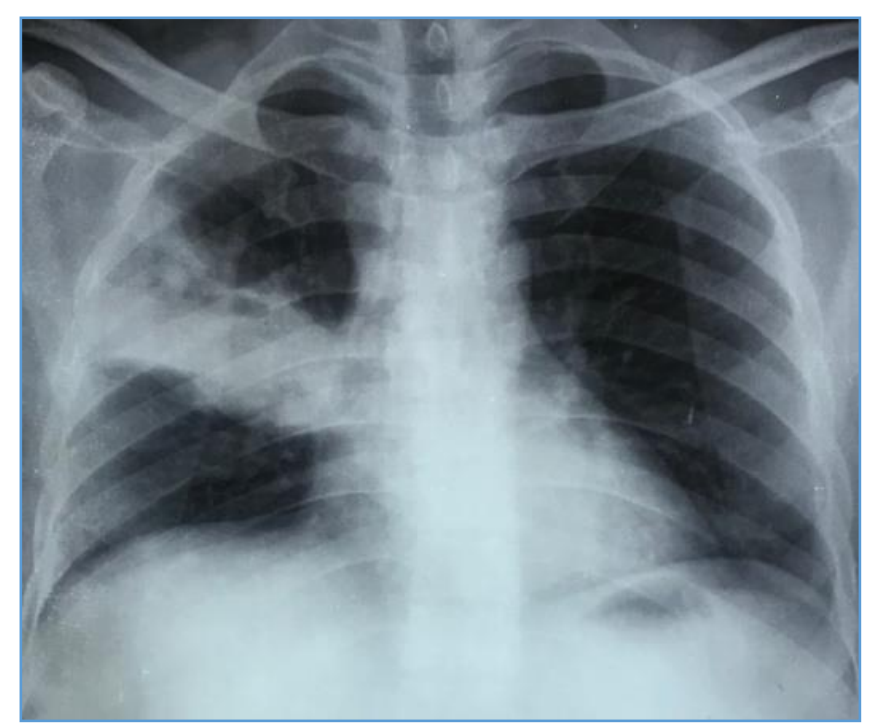

Figure 2. Chest radiograph-2

He was referred to our center, clinical assessment was done, documented pallor, heart rate-100/min, respiratory rate-20 breaths/min, $\mathrm{BP}-10 / 70 \mathrm{mmhg}$, Spo2-94\% at room air, respiratory system examination-crepitations in right infraclavicular and interscapualr area right side. Laboratory workup documented, as follows:
Hemoglobin-10 gm\%,

Total white blood cell counts- $18000 / \mathrm{mm}^{3}$,

Platelets-4.3 lakhs,

Blood sugar- fasting $230 \mathrm{mg} \%$,

Post prandial-390 mg\%,

HbA1C-12.8\%,

Creatinine-1.6 mg\%,

HIV-nonreactive, and

Liver functions and serum electrolytes were normal.

CT thorax has been done at our center sowing dense consolidation, with areas of lucency and breakdown or tiny cavitations in right upper lobe anterior segment; lucency is documented at the end points of pulmonary vasculature which may suggest underlying vasculitis or infarction (Figure 3). CT thorax image also suggests area of circular lucency occupying central opacity or haziness called as 'reverse halo sign' characteristic feature of invasive fungal infections (Figure 4).

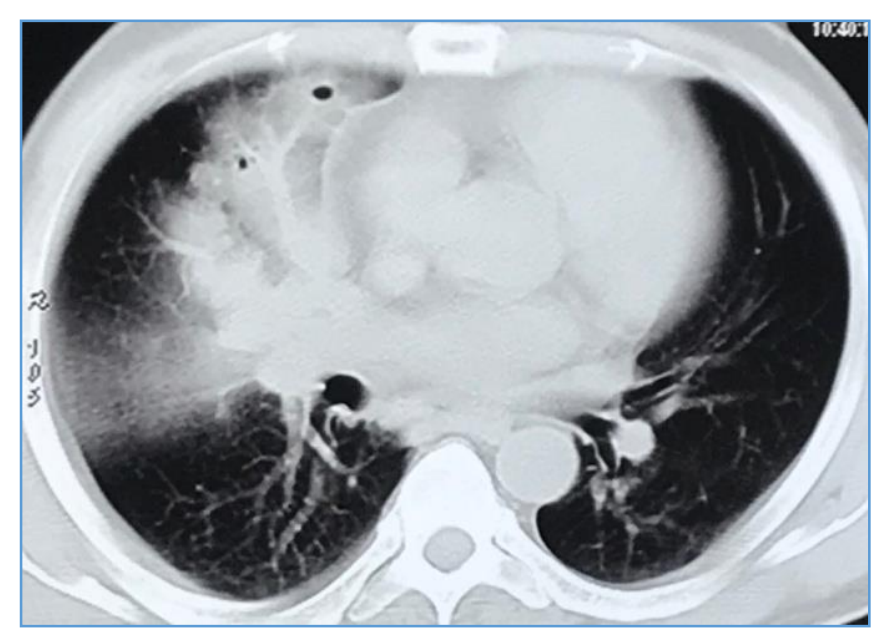

Figure 3. CT thorax-lucency with consolidation

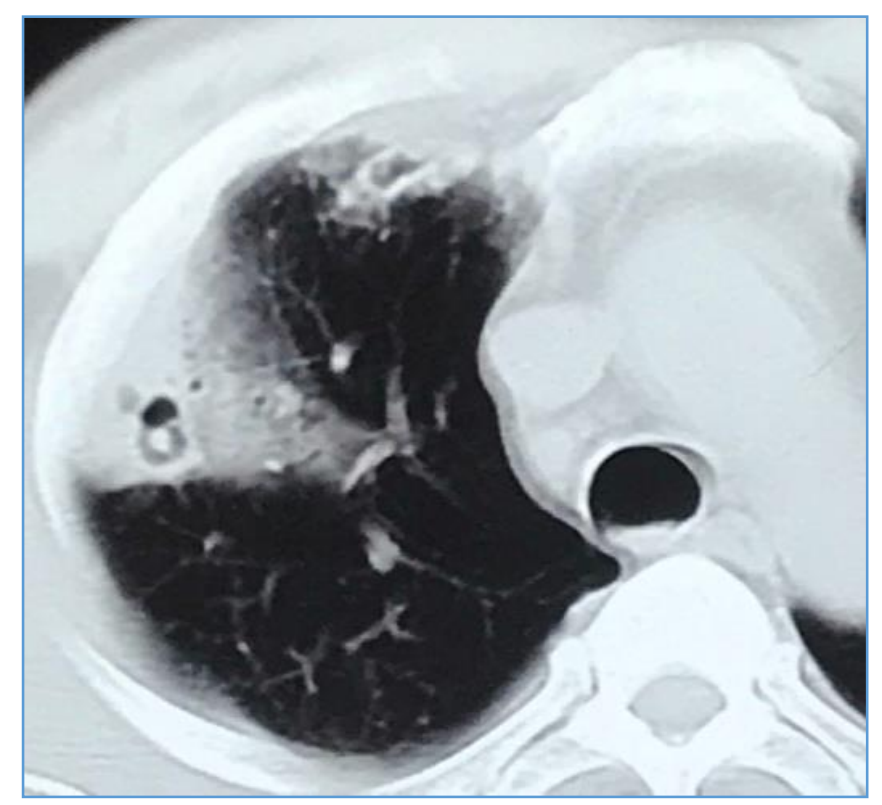

Figure 4. Reverse halo sign 
We have done fiberoptic videobronchoscopy and show themin Figure 5 and Figure 6.

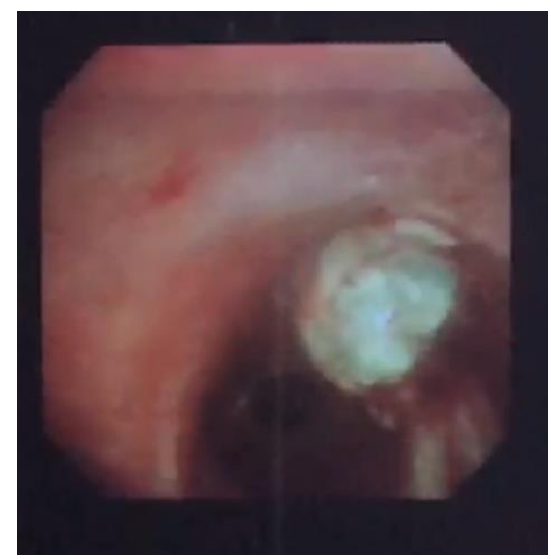

Figure 5. Cauliflower, fungating growth

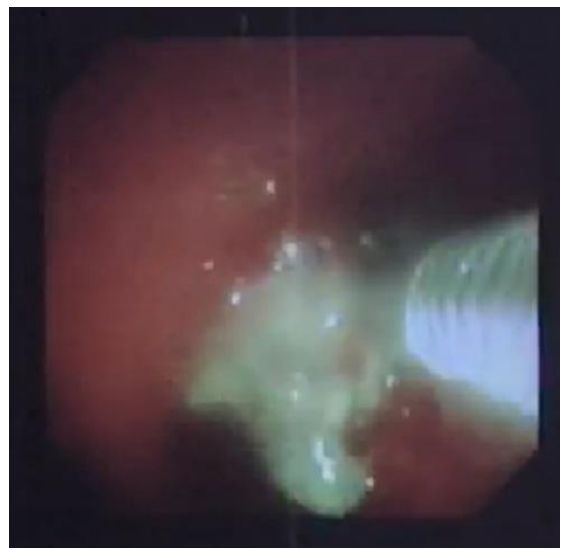

Figure 6. Forcep biopsy with fungating growth

\section{Biopsy Specimen}

During bronchoscopy we removed large cast like necrotic tissue $5 \mathrm{~cm}$ in length, collected in formalin container and sent for histopathology analysis (Figure 7). After removal of necrotic lesions, we documented massive endobronchial bleeding presenting as massive hemoptysis during bronchoscopy, and we have used topical adrenaline, topical botroclot and cold saline for control of bleeding.

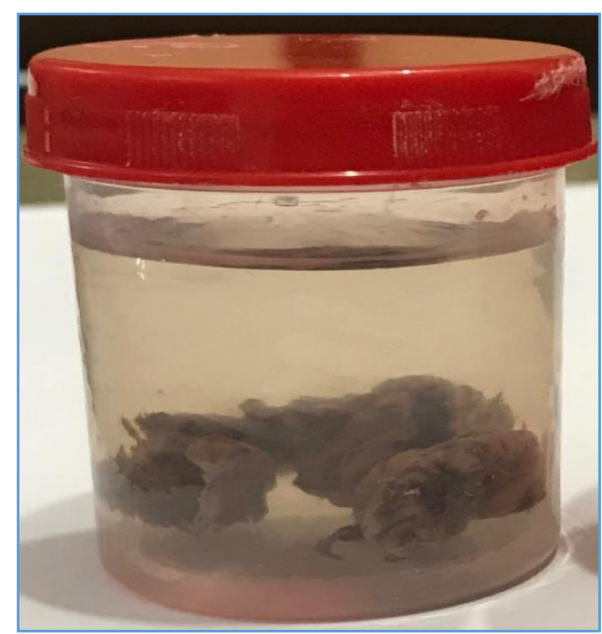

Figure 7. Cast like variegated specimen
Post bronchoscopy chest $\mathrm{x}$-ray repeated due to catastrophic endobronchial bleeding and to assess possibility of blood clots causing endobronchial obstruction, lung collapse and other post-obstructive complications. Chest x-ray was showing partial clearance of opacity of right upper lobe as we have removed 5-6 cm endobronchial mass during bronchoscopy (Figure 8).

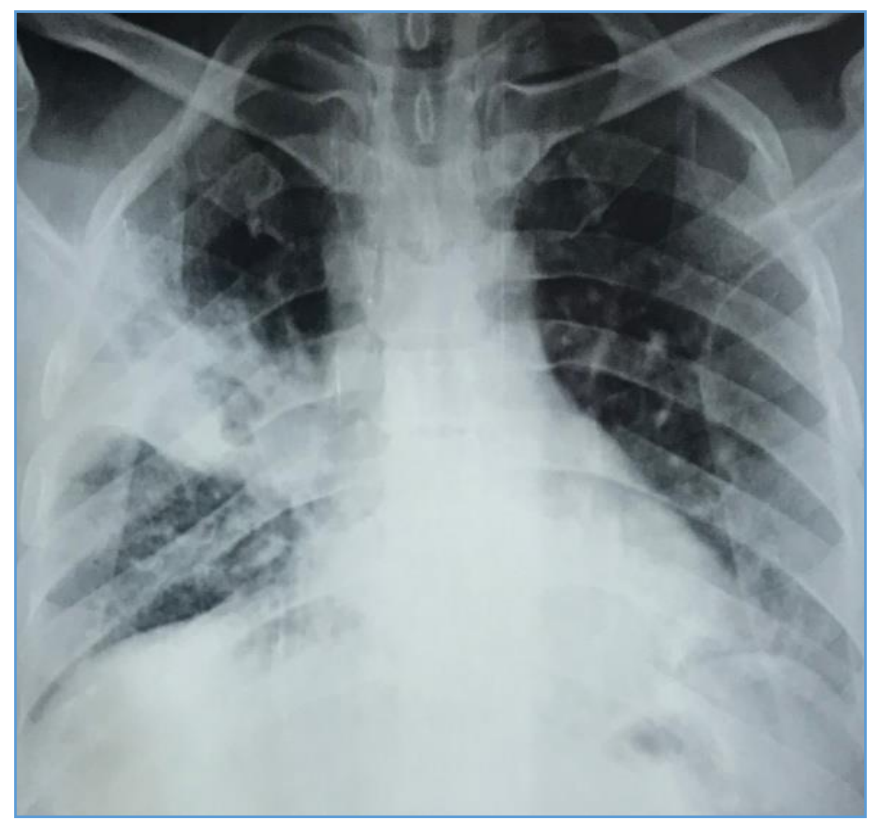

Figure 8. Chest radiograph-3

\section{BAL Study (Bronchoalveolar Lavage)}

Microscopy-smears show inflammation with karyorrhectic debris. Alveolar macrophages and squamous cells are also seen. Atypical cells are not apparent.

Gram stain-no organism seen,

Z-N stain-acid fast bacilli not seen, and

BAL cytology reveals neutrophil-rich inflammation.

\section{Bronchoscopic Forcep Biopsy Report}

GROSS: Bronchoscopic forcep biopsy-cast like necrotic tissue $5 \mathrm{~cm}$ in length.

MICROSCOPY: Sections of both tissue reveal presence of fungal infection causing extensive.

Parenchymal necrosis, angioinvasion and cartilage destruction along with bronchiolar invasion with neutrophilic and lymphocytic infiltrates documented. Organism is seen as broad, bulbous, aseptae hyphae, suggestive of invasive zygomycosis of right lung (Mucormycosis) (Figure 9, Figure 10, Figure 11, and Figure 12).

We have started Liposomal Injection Amphotericin B in dosage of $3 \mathrm{mg} / \mathrm{kg}$ per day in $250 \mathrm{ml}$ dextrose saline over 2hour infusion and adequate hydration maintained for renal protection give for 21 days, along with hemocoagulants like tranexamic acid and botropause injections for 5 days to prevent hemoptysis and catastrophic massive bleeding. We have monitored renal functions, hemoglobin and serum electrolytes, ECG for cardiac conduction abnormalities secondary to Amphotericin use. We have continued liposomal 


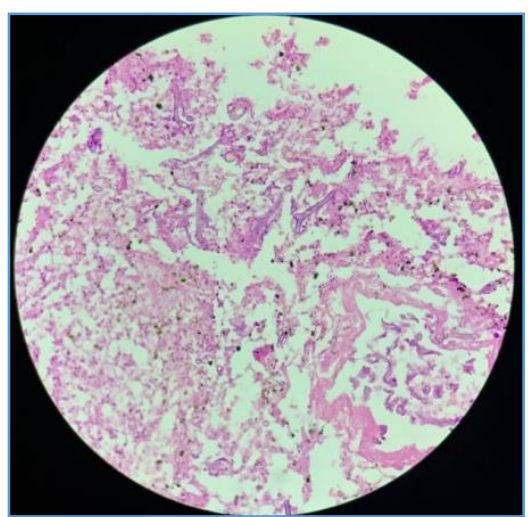

Figure 9. Histopathology-1

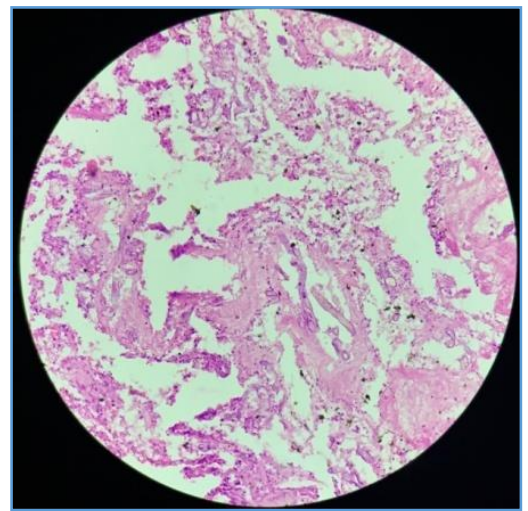

Figure 10. Histopathology-2

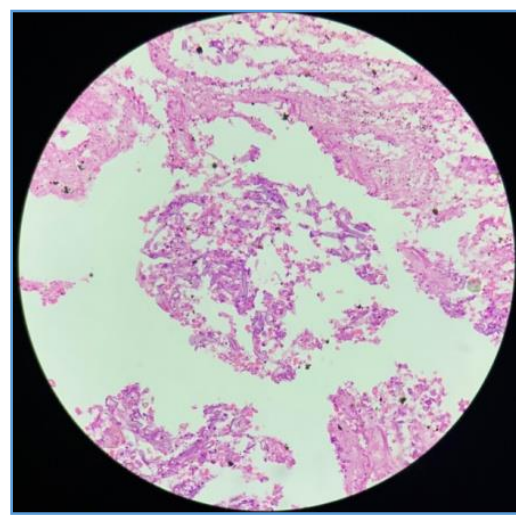

Figure 11. Histopathology-3

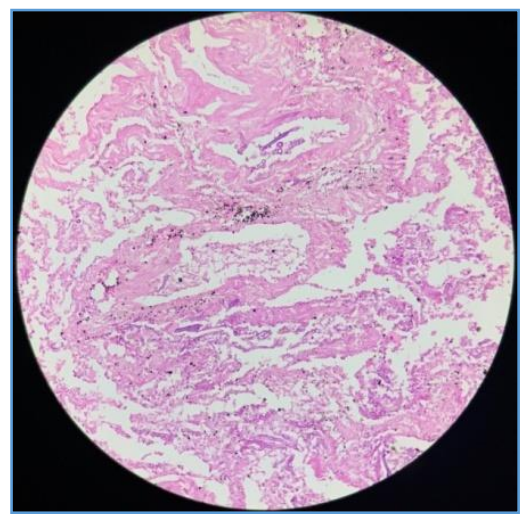

Figure 12. Histopathology-3
Amphotericin B for total 21 days. We have documented satisfactory compliance to Liposomal Amphotericin B with negligible side effects. We have repeated chest $\mathrm{x}$-ray at the completion of 21 days of treatment and documented significant radiological resolution in opacity of right upper zone (Figure 13). The patient was discharged on oral Posaconazole tablet $300 \mathrm{mg}$ daily for 12 weeks and we have monitored renal functions monthly and pulmonary lesions were clearly followed till 12 weeks.

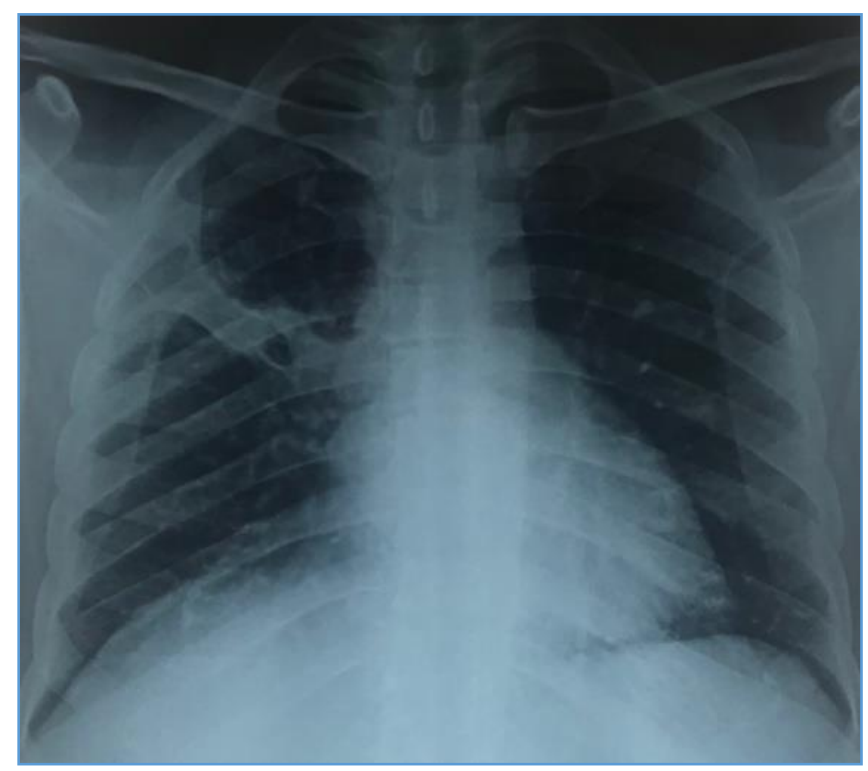

Figure 13. Chest radiograph-4

We have documented near complete resolution of pulmonary lesions in 12 weeks (Figure 14). We have advised for strict control of blood sugar during this follow up and also for strict follow up till 2 years to prevent recurrence.

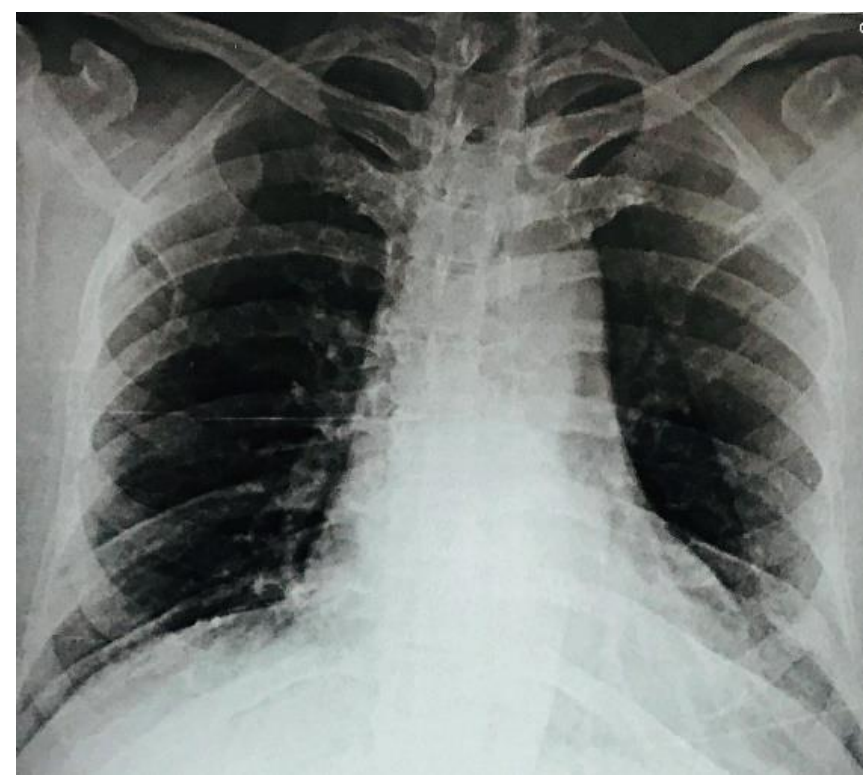

Figure 14. Chest radiograph-5 


\section{DISCUSSION}

Fungi of the Mucor genus are conditional pathogens that rarely cause disease in healthy individuals. However, inhalation of these spores may result in disease in subjects with impaired immune function. The organisms spread through the paranasal sinuses and respiratory system, or by the hematogenous or lymphatic route. The majority of patients with mucormycosis present one or more risk factors, including hematopoietic malignancies, treatment with immune inhibitors or diabetes mellitus [7]. Our patient was also having 'uncontrolled and undiagnosed Diabetes mellitus' as predominant risk factor for pulmonary Mucormycosis.

Pulmonary mucormycosis has an acute onset and a wide range of clinical manifestations, such as fever (most common), cough, hemoptysis, and dyspnea. Hemoptysis was found in about $25-30 \%$ of the patients. We have also documented recurrent, intermittent hemoptysis in our case. The clinical manifestations of pulmonary mucormycosis infection cannot be easily distinguished from those of pulmonary bacterial infection. As of our case, which was misdiagnosed as tuberculosis and received 4 months of anti-tuberculosis treatment before final diagnosis. Patients may present with fever, cough, expectoration, hemoptysis, and pleuritic chest pain. The laboratory examination results are nonspecific, with most patients only showing an increase in their WBC count. The radiographic presentations of patients with pulmonary mucormycosis appear abnormal and include infiltrate, cavity, consolidation, air crescent sign, pleural effusions, fistula, pneumothorax and pulmonary collapse [8].

In few patients, the disease manifests as airway obstruction as the fungus invades bronchus and forms intrabronchial lesions even though chest radiography shows mediastinal widening or pulmonary atelectasis. We have also documented as post-obstructive lung collapse secondary to endobronchial growth in our case. Bronchoscopy appears to be a successful diagnostic modality in diagnosing mucormycosis with major airway involvement. Bronchoscopy reveals granulation tissue and grey-white mucoid material that frequently blocks a major airway. The airways involved are typically edematous and necrotic, or lesions with an appearance suggestive of a bronchial adenoma. In many cases, it is postulated that a submucosal, invasive fungal infection caused a submucosal abscess, which presented as an endobronchial mass. [9] we have also documented similar bronchoscopy findings in our case. Reverse halo sign is a diagnostic radiological clue toward a diagnosis of Mucormycosis. We have also documented reverse halo sign in CT (computerized tomography) thorax study of our patient. As mucormycosis is an angioinvasive organism, it has a tendency to grow toward blood vessels, and a mass or nodule encroaching toward the great vessels of the mediastinum in successive radiographs suggests the possibility of mucormycosis. Similar findings of vasculitis and lung infarction was also documented in CT thorax as showing lucency at terminal points of lung parenchymal vasculature. The typical bronchoscopic findings are bronchial stenosis or obstruction, erythematous mucosa, gelatinous or mucoid secretions, fungating or polypoid masses and mucosal ulceration. The high degree of suspicion is essential as a noninvasive diagnosis with sputum culture is difficult to achieve [9]. We have done bronchoscopy interventions as near complete removal of endobronchial fungating mass lesion with forcep and cryoinstrument as we don't know as Mucormycosis as cause of central upper lobe endobronchial lesions and we have documented massive hemoptysis after procedure. Bleeding was controlled with topical hemocoagulants such as botroclot and cold saline and intravenous tranexamic acid injections.

Successful treatment of pulmonary mucormycosis relies on a timely diagnosis. Amphotericin B, along with surgical resection of the involved areas of the lung and treatment of the underlying disease, is the mainstay of treatment [10]. Despite the risk of renal toxicity, amphotericin B (1-1.5 mg/kg/day) remains the gold-standard antifungal agent used against mucormycosis. Oral posaconazole is also recommended, but these two types of drugs are often ineffective without surgical intervention [11]. Although the therapy duration is not well defined, a total cumulative dose of $1.5 \mathrm{~g}$ of amphotericin is usually sufficient in the selective group of patients who respond only to amphotericin therapy. We have documented satisfactory radiological response after 3-week duration of treatment with liposomal amphotericin and near complete radiological resolution at 3 months oral Posaconazole treatment without surgical interventions.

\section{CONCLUSIONS}

Key learning points from this case report can be listed, as follows:

1. Although recurrent hemoptysis is observed in tuberculosis, needs more workup in cases with similar clinical picture with minimal constitutional symptoms like fever, anorexia and weight loss.

2. Absence of clinical and radiological response mandates HRCT thorax as a protocol and chest radiology expertise has significant role in diagnosis and treatment of these cases.

3. 'Reverse halo sign' is important radiological sign which can be documented in various pathological conditions including invasive fungal infection like mucormycosis, and histopathology evidence is must before empirical antifungal treatment.

4. Bronchoscopy is important interventional procedure of choice in such cases to screen other causes like fungal infection and malignancy as cause for recurrent hemoptysis.

5. During Bronchoscopy, interventional pulmonologist should make all possible arrangements during Bronchoscopy to manage post-biopsy catastrophic bleeding as these lesions are necrotic and Hypervascular; and having propensity of massive bleeding post-procedure.

6. Invasive Bronchopulmonary Mucormycosis (IBPM) is difficult to treat, as many of these cases were having underlying poorly controlled diabetes mellitus and needs intensive sugar control with antifungal therapy for 14 to 21 days with renal functions monitoring, as 
these drugs have major concern of altered renal functions during treatment.

7. Although risk of recurrence has been documented in IBPM with poorly controlled diabetes, adequate antifungal treatment with intensive insulin treatment will have successful treatment outcome.

Author contributions: All co-authors have involved in all stages of this study while preparing the final version. They all agree with the results and conclusions.

Funding: No external funding is received for this article.

Declaration of interest: The authors declare that they have no competing interests.

Ethics approval and consent to participate: Not applicable. Availability of data and materials: All data generated or analyzed during this study are available for sharing when appropriate request is directed to corresponding author.

\section{REFERENCES}

1. Spellberg B, Maertens J. "Mucormycosis," in Principles and Practice of Transplant Infectious Diseases, A. Safdar, Ed., Springer, New York, NY, 2019. doi: 10.1007/978-1-4939-90344_34.

2. Pennell E, Pecson I, Nakamura C, Galvis AE. Pulmonary mucormycosis in an adolescent female with type 1 diabetes mellitus. IDCases, 2018; 14: e00474. doi: 10.1016/j.idcr.2018.e00474 PMid:30524955 PMCid: PMC6279882.

3. Serris A, Danion F, Lanternier F. Disease entities in mucormycosis. J Fungi, 2019; 5(1): 23. doi: 10.3390/jof5010023 PMid:30875744 PMCid:PMC6462957.

4. Luo Z, Zhang L. Diagnosis and treatment of pulmonary mucormycosis: a case report. Exp Ther Med, 2017; 14(4): 378891. doi: 10.3892/etm.2017.4986 PMid:29042980 PMCid: PMC5639346
5. Spellberg B, Edwards J, Ibrahim A. Novel perspectives on mucormycosis: pathophysiology, presentation, and management. Clin Microbiol Rev, 2005; 18(3): 556-69. doi: 10.1128/CMR.18.3.556-569.2005 PMid:16020690 PMCid: PMC1195964

6. Nam B, Kim TJ, Lee KS, Kim TS, Han J, Chung MJ. Pulmonary mucormycosis: serial morphologic changes on computed tomography correlate with clinical and pathologic findings. Eur Radiol, 2018; 28(2): 788-95. doi: 10.1007/s00330-017-5007-5 PMid:28812135.

7. Skiada A, Pagano L, Groll A, Zimmerli S, Dupont B, Lagrou K, Lass Florl C, Bouza E, Klimko N, Gaustad P, et al. Zygomycosis in Europe: Analysis of 230 cases accrued by the registry of the Europe confederation of medical mycology (ECMM) working group on zygomycosis between 2005 and 2007. Clin Microbiol Infect 2011; 17: 1859-67. doi: 10.1111/j.1469-0691.2010.03456.x PMid:21199154.

8. Lee FY, Mossad SB, Adal KA. Pulmonary mucormycosis: The last 30 years. Arch Intern Med. 1999; 159: 1301-9. doi: 10.1001/archinte.159.12.1301 PMid:10386506.

9. Husari AW, Jensen WA, Kirsch CM, Campagna AC, Kagawa FT, Hamed KA, et al. Pulmonary mucormycosis presenting as an endobronchial lesion. Chest 1994; 106: 1889-91. doi: 10.1378/chest.106.6.1889 PMid:7988219.

10. Mohammadi A, Mehdizadeh A, Ghasemi-Rad M Habibpour H, Esmaeli A. Pulmonary mucormycosis in patients with diabetic ketoacidosis: A case report and review of literature. Tuberk Toraks 2012; 60: 66-9. doi: 10.5578/tt.2464 PMid:22554371.

11. Brugière O, Dauriat G, Mal H, Marrash-Chalha R, Fournier M, Groussard $\mathrm{O}$, Besnard $\mathrm{M}$, Lesèche $\mathrm{G}$ and Dupont $\mathrm{B}$. Pulmonary mucormycosis (zygomycosis) in a lung transplant recipient: Recovery after posaconazole therapy. Transplantation 2005; 80: 1361-2. doi: 10.1097/01.TP.0000164140.26123.E6 PMid: 16382553 